\title{
Alterations in self-grooming sequences in the rat as a consequence of hippocampal damage
}

\author{
RICHARD L. CANNON, DANIEL J. PAUL, RONALD H. BAISDEN, \\ and MICHAEL L. WOODRUFF \\ James H. Quillen College of Medicine, East Tennessee State University, Johnson City, Tennessee
}

\begin{abstract}
The first purpose of the present experiments was to make a detailed study of the effects of hippocampal lesions on self-grooming behavior in rats. Hippocampal lesions decreased total grooming time and almost eliminated complete sequential grooming patterns, but increased open-field activity. Both hippocampal and neocortical lesions altered grooming in response to sucrose spray. The second purpose of this study was to compare the effects of the neurotoxin trimethyltin (TMT) with those produced by surgical destruction of the hippocampus. High doses of TMT, which produced extensive cell loss in the hippocampus, increased activity in the open field, but only moderate doses of TMT altered grooming. The results of the present study indicate that surgical removal of a large part of the hippocampus can disrupt a sequential, unlearned behavior. Furthermore, the behavioral effects of TMT cannot always be attributed to its neurotoxic action on the hippocampus.
\end{abstract}

The results of several experiments support the proposal that the hippocampus, in addition to its well-known involvement in mnemonic function, is involved in the normal expression of some species-typical behaviors that are either considered to be innate, or are acquired early in life before the animal suffers the lesion. For example, Nonneman and Kolb (1974) found that hippocampal lesions reduced both the response of cats to the odor of cat urine and affective responses of cats in social situations. Furthermore, rats with hippocampal lesions placed in pairs into an open field are significantly less likely than control rats to make contact with each other; hippocampal lesions also eliminate shock-induced aggression in these animals (Kolb \& Nonneman, 1974).

The effects of hippocampal lesions are not restricted to alterations in response to cospecifics. Hippocampal lesions in both rats (Woodruff \& Bailey, 1979) and rabbits (Woodruff, Hatton, \& Meyer, 1975) prolong the defensive immobility response that often follows release from physical restraint (Gallup, 1974). Additionally, several reports indicate that hippocampal lesions may decrease some measures of the self-grooming that occurs in rats when they are placed into a novel environment such as an open-field arena (Bär, Gispen, \& Isaacson, 1981; Oades \& Isaacson, 1977; Reinstein, Hannigan, \& Isaacson, 1982; Vanderwolf, Kolb, \& Cooley, 1978).

These experiments support the hypothesis that hippocampal lesions decrease self-grooming in rats. However, three other experiments failed to find any effect of hippocampal lesions on grooming in rats (Colbern, Isaacson,

This research was supported by NIH Grant ES 04070-05 to MLW. Requests for reprints should be addressed to $R$. L. Cannon, Department of Anatomy, P.O. Box 70,582, James H. Quillen College of Medicine, East Tennessee State University, Johnson City, TN 37614.
Bohus, \& Gispen, 1977; Hannigan, Springer, \& Isaacson, 1984; Kolb \& Nonneman, 1974). An examination of the methods used to study grooming in these various studies suggests that the reason for the apparent discrepancy in results may rest in the observational methods used. Most of the studies employed some form of "time sampling" procedure to monitor grooming. This procedure involves observing a rat's behavior only at given time intervals and simply recording whether or not the rat is exhibiting some component of grooming behavior. This permits the experimenter to miss instances of occurrence of the behavior, or, conversely, might inflate the grooming score if, by chance, the rat was grooming at several observational time points. Also, the timesampling method does not yield information regarding the frequency and sequence of individual components of the grooming sequence. Therefore, the first purpose of the experiments reported in this paper was to determine the effect of aspiration hippocampal lesions on grooming in rats by using continuous observation and scoring of the several components that constitute the chain of behaviors that comprise the normal grooming sequence in the rat.

The effects of the neurotoxin trimethyltin (TMT) on grooming were also investigated here. It is generally noted that TMT produces its major neurotoxic effect on the neurons of the hippocampus (Brown, Aldridge, Street, \& Verschoyle, 1979; Chang, 1986; Dyer, Deshields, \& Wonderlin, 1982; McMillan \& Wenger, 1985) and also produces effects on locomotor activity and measures of learning and memory that are similar to those produced by surgical destruction of the hippocampus (Bushnell, 1990; McMillan \& Wenger, 1985; Woodruff, Baisden, Whittington, Shelton, \& Wray, 1988). However, with the exception of activity measures and acute effects of the toxin, such as tail mutilation (Dyer, Walsh, Wonderlin, 
\& Bercegeay, 1982), little information is available concerning the effect of TMT on unlearned behavioral patterns. Therefore, the second purpose of the present study was to examine the effects of TMT on self-grooming in rats. Additionally, locomotor activity in an open field was examined to provide a parallel to previous research in which either surgical or TMT-induced damage to the hippocampus increased activity in an open field (McMillan \& Wenger, 1985; O'Keefe \& Nadel, 1978).

\section{METHODS}

\section{Subjects}

Eighty male Long-Evans rats weighing between 225 and $255 \mathrm{~g}$ were housed individually in a temperature-controlled facility on a 12:12-h light:dark cycle. Lights were turned on at $0700 \mathrm{~h}$ and testing was conducted between 1300 and $1600 \mathrm{~h}$. After recovery from treatment procedures, the animals were gradually reduced to $85 \%$ of their ad-lib weight and tested in an operant discrimination prior to the observations. Food and water were available ad lib both during recovery from the procedures and after the operant training was concluded.

\section{Surgical Procedures}

For Experiment 1, 10 rats received bilateral aspiration lesions of the hippocampus (Group HL) and overlying dorsolateral neocortex, and 10 rats were given lesions restricted to the neocortex (Group CL). The lesions were made using procedures typically used in this laboratory (e.g., Isaacson \& Woodruff, 1975). Surgical anesthesia equivalent to State II, Plane 2 in clinical application (Collins, 1976, pp. 253-264) was induced by an injection of sodium pentobarbital (Nembutal, $50 \mathrm{mg} / \mathrm{kg}$, i.p.) and was supplemented with an intramuscular injection of an acepromazine $(2 \mathrm{mg} / \mathrm{kg}) / \mathrm{keta}$ mine hydrochloride $(\mathbf{4 0} \mathrm{mg} / \mathrm{kg})$ cocktail, if necessary to maintain anesthesia. The scalp was then shaved and the rat was placed in a Kopf stereotaxic instrument. A midline incision was made in the scalp. The scalp and the underlying muscles and fascia were retracted. The dura over the dorsolateral sensorimotor cortex was exposed by bilateral burr holes. The dura was reflected and the appropriate amount of brain tissue was removed by aspiration, using a 24-ga blunt hypodermic tube. After hemostasis was achieved, the scalp was sutured and a prophylactic injection of penicillin $\mathbf{G}$ benzathine and penicillin $G$ procaine (G. C. Hanford, 3,000 units, i.m.) was given. A third group of rats served as nonoperated controls (Group N).

\section{Trimethyltin Administration}

For Experiment 2, 50 rats were randomly assigned to one of five equal-sized groups. The rats in the four TMT-treated groups were lightly anesthetized with ether and orally gavaged with TMT chloride (Alfa Products), which was dissolved in saline for effective doses of $2,4,6$, or $7 \mathrm{mg} / \mathrm{kg}$ (expressed as the free-base weight), immediately prior to treatment. The remaining 10 rats were lightly anesthetized and gavaged with an equivalent volume of saline to become a treatment control group. An average of 120 days elapsed between the surgery or TMT dosing and the behavioral observations reported here.

\section{Apparatus and Procedures for Behavioral Testing}

Activity and grooming were observed by two investigators. A video camera suspended over the test field allowed the observations to be made from an adjacent room on a color monitor. In both experiments, the rats were first observed for three consecutive 15 min daily sessions in an open field constructed of black Plexiglas and measuring $91.5 \times 91.5 \times 34.5 \mathrm{~cm}$. White lines painted on the floor of the field created 36 equal-area squares. The number of line crossings and rearings were recorded by one investigator using a pushbutton that advanced an electromechanical counter when it was depressed. Additionally, solid-state timers, gates, and steppers advanced a bank of counters one unit every $90 \mathrm{sec}$ so that within-session changes in activity could be monitored. A line crossing was counted when the rat placed both front paws and at least $50 \%$ of its body across a line. A rearing was counted when a rat raised up into a vertical position and did not emit any grooming component.

The second investigator recorded grooming responses by using software written in Turbo Basic (Version 1.0, Borland International Inc.) for an IBM/PC/AT-compatible microcomputer. The time between being placed in the open field or being sprayed with a sucrose solution, as described below, and emission of the first grooming response was recorded, as were the total time spent grooming and the total number of grooming episodes. Additionally, grooming components were individually recorded in the sequence in which they occurred and the number of individual grooming components in each grooming episode were tallied. The grooming components were: rubbing the paws together; washing the face with the paws and upper forelegs; rubbing the head outside of the facial mask with the paws and upper forelegs; grooming the right or left side of the body with the mouth; grooming the abdomen with the mouth; grooming the anogenital region with the mouth; scratching; grooming the tail; shaking the paws; or shaking the body.

The normal grooming pattern for the rat presents a cephalocaudal sequence, which includes many of these components in a given grooming episode (Richmond \& Sachs, 1980). To quantify the type of grooming patterns exhibited by each rat, the sequence of grooming components presented in each episode was classified as comprising either a complete or fragmented chain of grooming components based on a four-phase system adapted from that used by Berridge (1989). Using these criteria, a complete chain would include the following sequence of phases. Phase 1 consists of rapid elliptical movements of the paws over the nose, vibrissae, and mouth. In Phase 2, unilateral or bilateral movement of the upper forelegs over the face area, from just above the eyes forward to the nose, occurs. In Phase 3, the forearm movement progresses to behind the ears and then down to the nose. Phase 4 consists of a transfer of cleaning to the body surface. A complete chain had all four phases in the order just described. A fragmented chain was scored if the rat began grooming but did not show all four phases in that episode, or if the phases were not in order (e.g., grooming the right side of the body, followed by movement of the paws over the nose, followed by body grooming the left side of the body, etc.).

Following the 3 days in the open field, grooming was observed for 3 consecutive days for $15 \mathrm{~min} /$ day in a clear plastic cage $(45.7 \times$ $24.1 \times 20.3 \mathrm{~cm})$. The rat was placed into the cage and subjected to 10 quick sprays of a $16 \%$ (w/v in distilled water) sucrose solution to elicit grooming (Moore, Low, \& Kulkosky, 1987). Data concerning grooming were collected in the same way as they had been in the open field.

\section{Histology}

Following behavioral observations, the rats that had been given lesions or TMT were sacrificed with an overdose of Nembutal and intracardially perfused with $0.9 \%$ saline, followed by $10 \%$ buffered formalin. The brains were removed, embedded in Paraplast, and sectioned in a coronal plane at $10 \mu \mathrm{m}$. Every 10 th section was slidemounted and stained with either thionin or hematoxylin and eosin.

Descriptions of the lesions within the brains of the rats from Groups HL and CL and an evaluation of the amount of damage done by the TMT were made using a Leitz Diaplan microscope. The Optimas image-analysis system was used to count neuron cell bodies in the medial frontal cortex, pyriform cortex, amygdala (central 
and medial nuclei), granule cell layer of the dentate gyrus, and fields CA1 and CA3 of Ammon's horn. TMT-induced neuropathology has been reported in each of these regions (e.g., Balaban, O'Callaghan, \& Billingsley, 1988) and, of the brain regions damaged by TMT, neuron loss in these areas may affect the behaviors studied (e.g., Isaacson, 1982). The cell counts were made from two sections through each structure. Photomicrographs of representative sections for each of the regions used for cell counts are presented in Figure 1. The total area delineated for cell counts for each structure was $50 \times 10^{5} \mu \mathrm{m}^{2}$ in the pyriform cortex, amygdala, dentate gyrus, and Ammon's hom. Layers II-VI were included in the counts for the neocortical regions. Only the granule cell and pyramidal cell layers were included in counts for the dentate gyrus and Ammon's horn, respectively.

\section{Statistical Analyses}

The activity and grooming data were analyzed for group, days, and interaction effects using a two-factor analysis of variance (ANOVA) with a repeated measure on the days factor. The ANOVA was also used to evaluate the effects of TMT on the number of neuron soma counted in the rats in the four treated groups and from 4 normal rats. The numbers were combined for regression analyses, since $t$ tests indicated no difference between the two sections from each region. Multiple comparison tests were applied post hoc when justified by significant $p$ values $(<.05)$ for the group effect.
Regression analyses were also run by using the cell counts obtained from the various anatomical regions, described above, as predictors of the behavioral effects.

\section{Experiment 1}

Activity. The activity data were analyzed both as the total number of lines crossed by each rat for each of the 3 days of testing and as the number of lines crossed in each 90 -sec period within each day by each rat. The ANOVA indicated significant group $[F(2,27)=10.82$, $p<.01]$ and days $[F(2,54)=7.98, p<.01]$ effects for the 3 days of testing, but the groups $\times$ days interaction was insignificant. Multiple comparison tests indicated that Group HL differed significantly from Group N on each of the 3 days, but only differed from Group CL on the second and third days of activity measurement (see Figure 2A).

Significant group $[F(2,27)=5.84, p<.01]$, time interval $[F(9,243)=28.37, p<.01]$, and interaction $[F(18,243)=4.69, p<.01]$ effects were found when

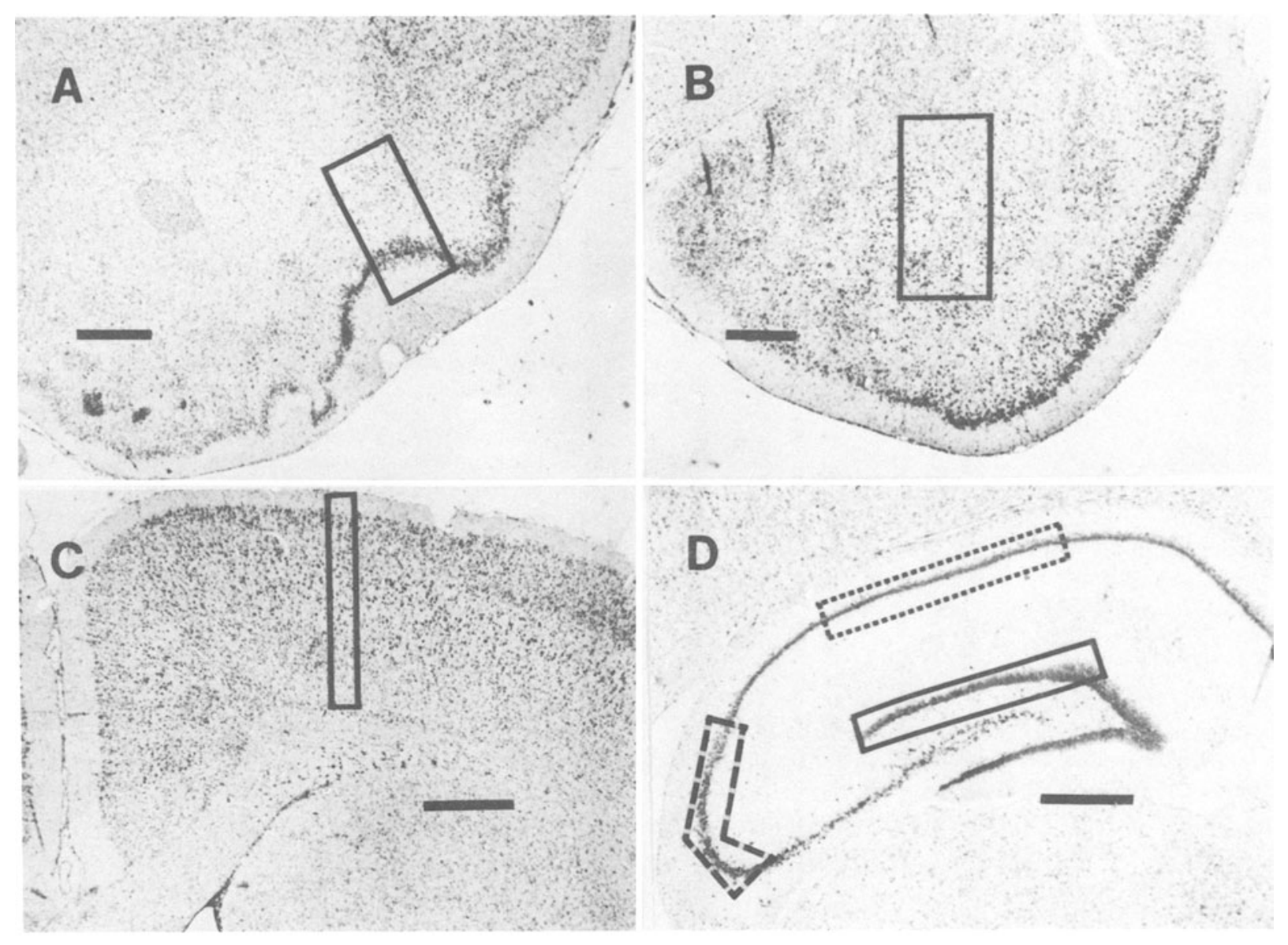

Figure 1. Cell counts were taken from the regions of pyriform cortex (A), amygdala (B), hippocampus (C), and medial frontal cortex (D), as indicated by the outlined areas in the photomicrographs. All sections were stained with thionin. Magnification bars are: $\mathrm{A}=$ $500 \mu \mathrm{m}, B=400 \mu \mathrm{m}, C=600 \mu \mathrm{m}$, and $D=500 \mu \mathrm{m}$. 

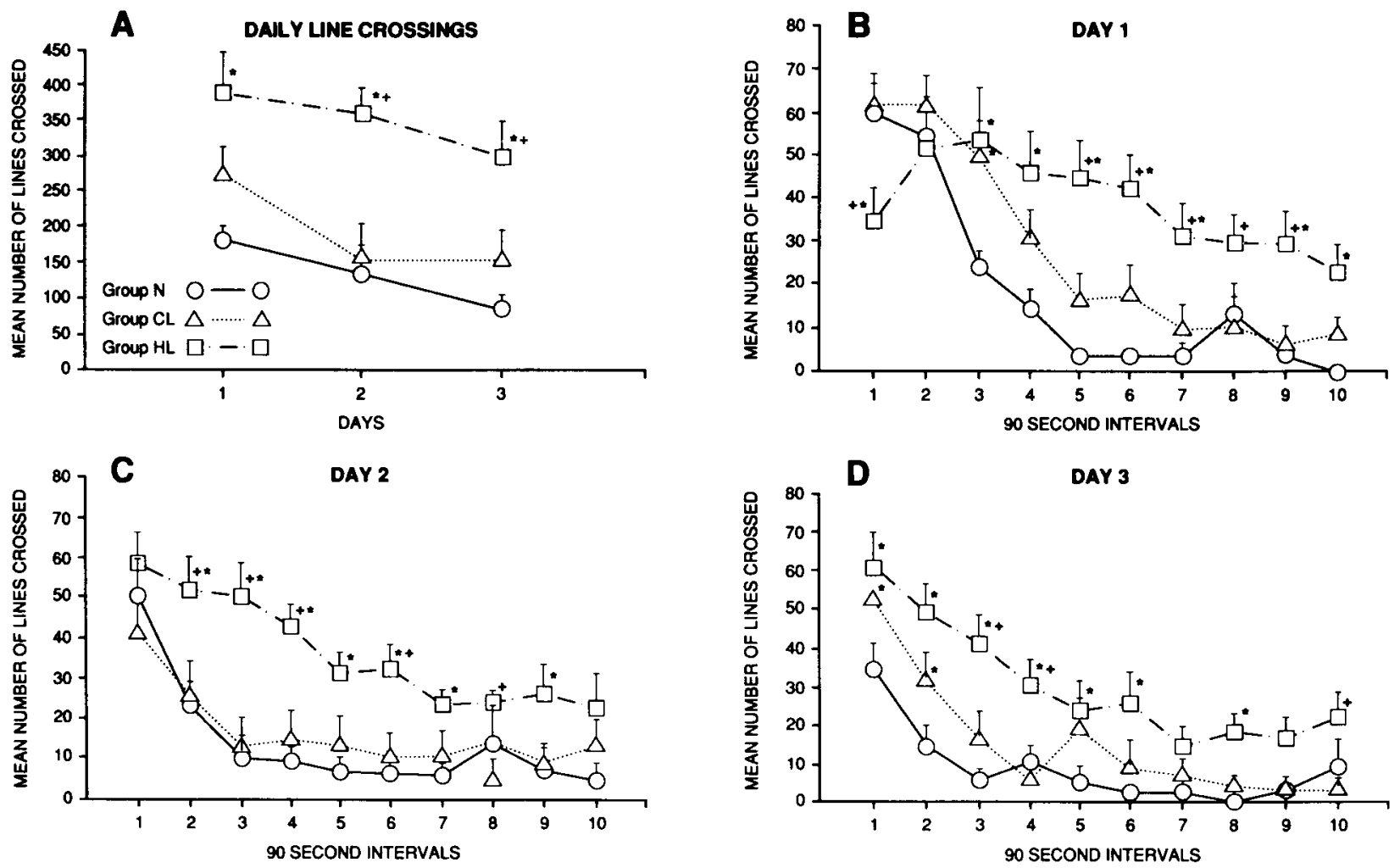

Figure 2. (A) Mean and standard error of the mean number of lines crossed by each group during each 15-min open-field observation period. Although Group CL never differed significantly from Group $\mathbf{N}$ in overall line crossing, a slight increase in activity was observed in this group early in testing and an analysis of within-sessions activity patterns revealed some significant differences between these groups (see panels B and D). * = differs from Group $N(p<.05) ;+=$ differs from Group CL $(p<.05)$. During the first 90 sec of Day 1 (B), the rats with hippocampal lesions were significantly less active than either of the control groups, but increased their activity during the second period and did not decrease activity levels as much as the controls across the remaining time intervals. The rats with cortical lesions were slightly more active than the normal controls during the first half of the first test session. This difference only achieved statistical significance during the third 90-sec interval. The performance of Group CL was virtually identical to that of $\mathrm{Group} N$ throughout Day 2 of testing (C), whereas Group HL continued to be more active for most of the session. Group CL exhibited a slight, but statistically significant, increase in activity during the first 3 min of testing during Day 3 (D), whereas the rats in Group HL were consistently more active than normal controls throughout the session. Symbols are shown in panel $A$.

the ANOVA was applied to the number of line crossings on Day 1. As can be seen in Figure 2B, the activity levels for Groups $\mathbf{N}$ and $\mathrm{CL}$ decreased substantially across the 10 time intervals, whereas those for Group HL did not. The results of post hoc multiple comparisons reinforced this observation. Group HL differed from Group $\mathrm{N}$ at time intervals $1,3,4,5,6,7,9$, and 10 . It is interesting that Group HL crossed significantly fewer lines during the first $90 \mathrm{sec}$ of Day 1 than either Group N or Group CL. It should also be noted that, whereas the mean total number of lines crossed on Day 1 did not differ between Groups $\mathrm{N}$ and $\mathrm{CL}$, the Group CL rats were slightly more active during the middle time intervals; this difference was statistically significant during the third 90 -sec interval (Figure 2B).

Significant group $[F(2,27)=8.23, p<.01 ; F(2,27)=$ $7.12, p<.01]$ and time interval $[F(9,243)=17.62, p<$ $.01 ; F(9,243)=23.3, p<.01]$ effects were found for Days 2 and 3 , but the interaction terms were not significant. As can be seen in Figures $2 \mathrm{C}$ and 2D, Group HL crossed significantly more lines than Group $N$ or Group $\mathrm{CL}$ during the majority of the time intervals on Days 2 and 3 , but the hippocampus-damaged rats did exhibit substantial within-session decreases in activity.

The results of the ANOVA applied to the number of rearings exhibited by each group each day yielded an insignificant group effect $[F(2,27)=0.82, p<.05]$. However, the days effect was significant $[F(2,54)=15.17$, $p<.01]$. The number of rearings declined over the 3day period; the interaction effect was not significant.

Grooming in the open field. The mean latency to exhibit some component of grooming after the rat was placed in the open field did not differ significantly among groups, but latency to groom decreased substantially and equally across days for all groups, as was indicated by a significant days effect $[F(2,54)=3.57, p<.05]$ and a nonsignificant interaction.

The total number of grooming episodes (both fragmented and complete) did not differ among groups and neither the days nor the interaction terms reached puta- 
tive levels of statistical significance for this measure. Hippocampal lesions did have some effect on total time spent grooming in the open field. A significant group effect was found $[F(2,27)=3.51, p<.05]$ and subsequent multiple comparison tests indicated that the rats in Group HL groomed for significantly less time on Days 1 and 2 than did the rats in Group $\mathrm{N}$ (see Figure 3A). However, Group HL did not differ from Group CL, which did not differ from Group N. No significant differences were found for Day 3 and neither the days nor the interaction effect was significant.

Hippocampal lesions had a greater impact on number of complete grooming sequences than on total time spent grooming. The group effect was very significant $[F(2,27)=$ $11.24, p<.01]$ and multiple comparison tests indicated that Group HL exhibited significantly fewer complete grooming sequences than either Groups $\mathrm{N}$ or $\mathrm{CL}$ on all 3 days of testing in the open field (Figure 3B). Group N
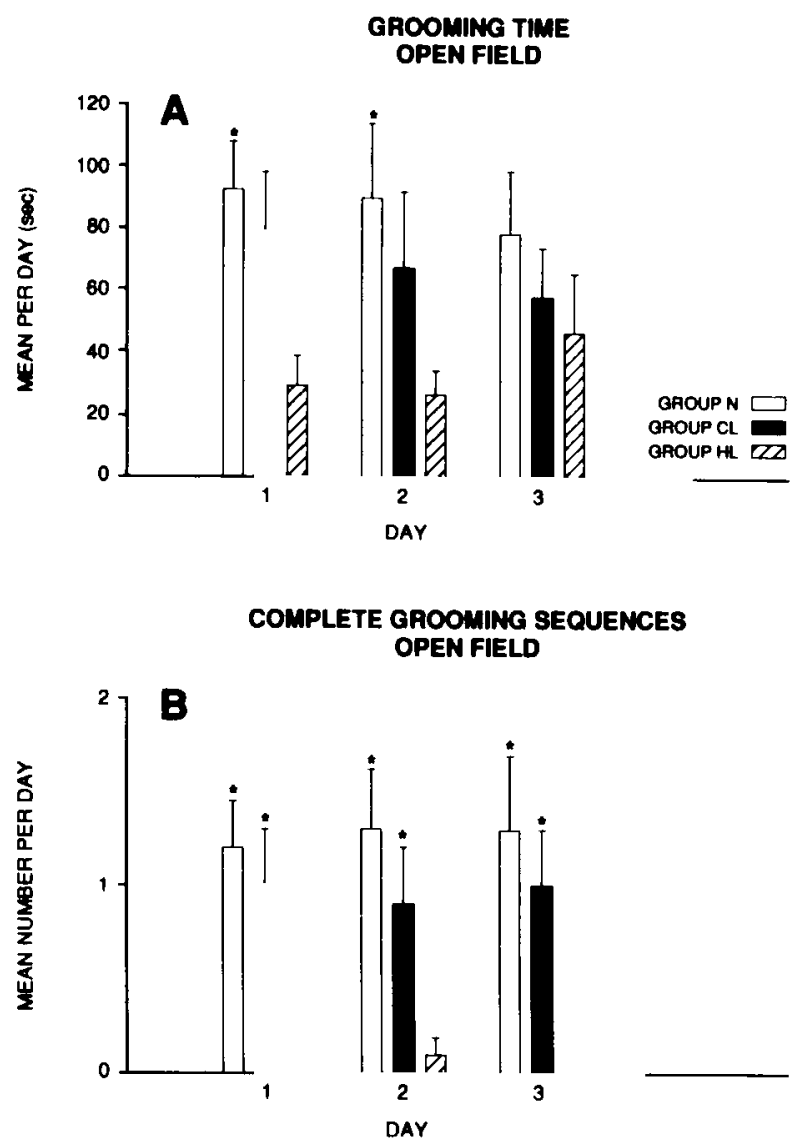

Figure 3. (A) Hippocampal lesions produced a decrease in total grooming time in the open field, but the effect was sufficiently variable within Group HL that it only differed significantly from Group $\mathrm{N}$ during the first 2 days of observation. Additionally, damage to the neocortex may have contributed to the effect, because Group CL never differed from Group HL. (B) Hippocampal lesions did have a very significant impact on the number of complete grooming sequences emitted in the open field, because no rat in Group HL exhibited a complete grooming sequence on Days 1 or $3 . *$ = differs from Group HL $(p<.05)$. did not differ from Group $C L$ and neither the days nor the interaction effect was significant.

The results of the ANOVAs applied to the data for time spent grooming and number of complete grooming sequences emitted during open-field testing indicate that the rats with hippocampal lesions groomed less than the control rats. These rats were also more active in the open field and the decreased grooming response might be a consequence of more time spent moving. If this were the case, negative correlations would be expected between grooming time and activity. However, when $r^{2}$ was calculated between time spent grooming and lines crossed or number of complete grooming sequences and lines crossed, no significant correlations were obtained. Indeed, the largest $r^{2}$ (.26) value for Group HL was obtained on Day 3, when this group did not differ from the controls.

Additionally, no significant effects were found when the repeated measures ANOVA was conducted on the number of fragmented grooming sequences emitted by each group, each day, in the open field. Because the number of complete grooming sequences and the number of fragmented grooming sequences together comprised the total number of grooming episodes, the lack of significance for number of fragmented grooming episodes explains the lack of significant group differences for total grooming episodes. Finally, no significant differences were found when the numbers of grooming components per episode, per group, were analyzed.

Grooming after spray. The effects of hippocampal lesions on spray-induced grooming differed in several respects from the effects of the lesions on grooming in the open field. First, a significant group effect was found when the latency to exhibit a grooming component after being sprayed was analyzed $[F(2,27)=6.38, p<.01]$ and multiple comparison tests indicated that Group HL had significantly longer latencies to initiate grooming on Days 2 and 3 of testing than either Group $\mathbf{N}$ or Group CL (see Figure 4A). No significant days or interaction effects were found.

Second, a significant group effect was found when a repeated measures ANOVA was applied to total time spent grooming after being sprayed $[F(2,27)=6.44, p<.01]$. The days and interaction effects were not significant. Multiple comparison tests indicated that Group CL groomed significantly longer than Group HL on all 3 days of testing and longer than Group $N$ on Day 1, whereas Group HL groomed for a significantly shorter period of time than Group N on Day 2 (Figure 4B). A significant group effect was also obtained for total number of episodes of grooming in the spray condition $[F(2,27)=6.71$, $p<.01]$ and, as was the case for total grooming time, the rats with cortical lesions differed significantly from Group N on all 3 days of testing. The rats in Group HL differed significantly from Group $\mathbf{N}$ on Day 3 (Figure $4 \mathrm{C}$ ). Multiple comparison tests failed to reveal any significant differences between Groups $\mathrm{HL}$ and $\mathrm{CL}$. Neither the days nor the interaction effect was significant.

Unlike their effect on grooming in the open field, hippocampal lesions had essentially no effect on the number 

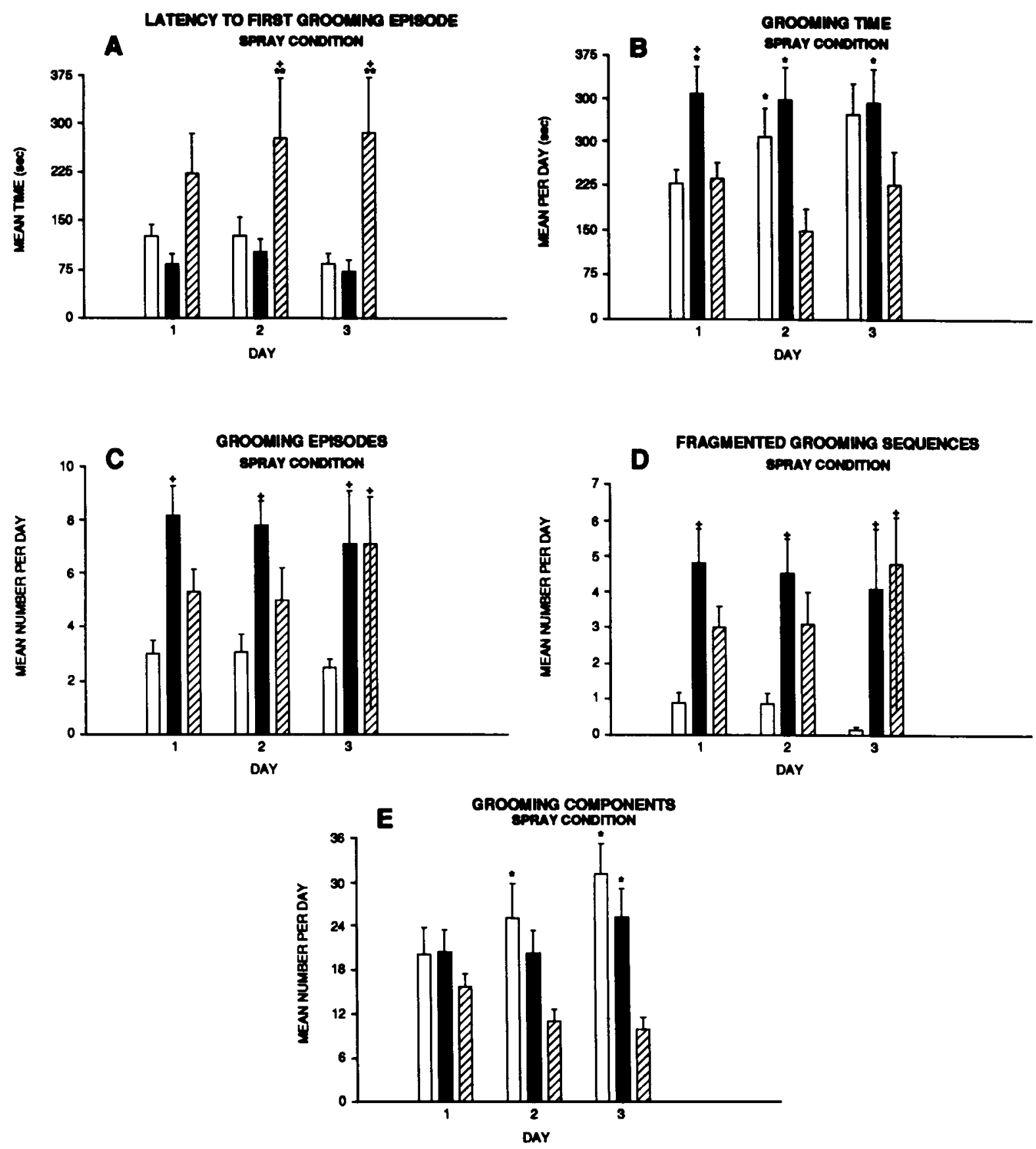

Figure 4. The effects of lesions on grooming elicited by the sucrose solution differed in several respects from those observe in the open field. The rats in Group $\mathrm{HL}$ took significantly longer to initiate grooming after being sprayed $(A)$ and exhibited fewi grooming components (E) during Test Days 2 and 3. However, lesions restricted to the neocortex significantly increased groomir time (B), number of grooming episodes (C), and number of fragmented grooming sequences (D), as compared with those measur in normal controls. $+=$ differs from Group $N(p<.05) ; *=$ differs from Group HL $(p<.05) ; * *$ differs from Group $C$ $(p<.05)$. 
of complete grooming episodes in the spray condition. Although a significant group effect was found $[F(2,27)=$ $3.63, p<.05]$, the only significant difference indicated by multiple comparison tests was between Groups $\mathrm{HL}$ and $\mathrm{CL}$ on the second day of testing. However, both cortical and hippocampal lesions significantly increased the number of fragmented grooming episodes after spray (Figure 4D), as was indicated by a significant group effect $[F(2,27)=7.15, p<.01]$. Multiple comparison tests showed that Group CL differed from Group $\mathrm{N}$ on all 3 days of testing and that Group HL differed from Group N on the third day. No other significant effects were found for this measure.

A significant group effect was also found for the number of components per grooming episode $[F(2 ; 27)=$ $6.96, p<.01]$. The days and interaction effects were not significant. Post hoc multiple comparison tests indicated that Group HL exhibited significantly fewer components per episode than the normal rats on the second and third days of testing, and fewer episodes than Group CL on the third day (Figure 4E).

Histology. An examination of the sections from the brains of the rats in Groups $\mathrm{HL}$ and $\mathrm{CL}$ indicated that the lesions in these animals were similar to those described in detail in previous communications (e.g., Woodruff, Baisden, Whittington, \& Benson, 1987). The temporal portion of the hippocampus was spared in all the rats in Group HL, as was the medial part of its septal (dorsal) extension. In all the rats, damage to structures other than the hippocampus included the neocortex and the corpus callosum overlying the hippocampus. In 4 rats, unilateral minor direct damage was also found to the dorsal aspect of the lateral thalamic nucleus. The observed behavior of these animals did not differ from the others in Group HL and their data was included in the analyses reported above.

The lesions in the rats in Group CL were restricted to the parietal cortex overlying the middle part of the anterioposterior extent of the hippocampus. They extended from approximately $3 \mathrm{~mm}$ posterior to bregma (as estimated from comparing the appearance of the hippocampus and thalamus to plates in the atlas of Pellegrino, Pellegrino, \& Cushman, 1979) caudally for between 2 and $3 \mathrm{~mm}$. In the medial lateral plane, the lesions began about $3 \mathrm{~mm}$ lateral to the midline and continued laterally for about $3 \mathrm{~mm}$. The corpus callosum was transected bilaterally in every rat in Group CL and the dorsal CA1 subfield of Ammon's horn was damaged unilaterally in 2 rats and bilaterally in 1 rat in this group. The behavioral data from these rats were within the range of rats in Group CL without hippocampal damage and were included in the analyses reported above.

\section{Experiment 2}

Activity. The mean total lines crossed for each day for each group is presented in Figure 5A. Significant group $[F(4,45)=10.07, p<.01]$ and days effects $[F(2,90)=$ $20.02, p<.01]$ were found. The interaction effect was not statistically significant. Multiple comparisons indicated that the group that was given $6 \mathrm{mg} / \mathrm{kg}$ of TMT (Group 6MG) and the group that was given $7 \mathrm{mg} / \mathrm{kg}$ of TMT (Group 7MG) differed significantly from all other groups each day, except for the lack of a statistically significant difference on Day 2 of testing between Group 7MG and the groups that were given $2 \mathrm{mg} / \mathrm{kg}$ (Group 2MG) and $4 \mathrm{mg} / \mathrm{kg}$
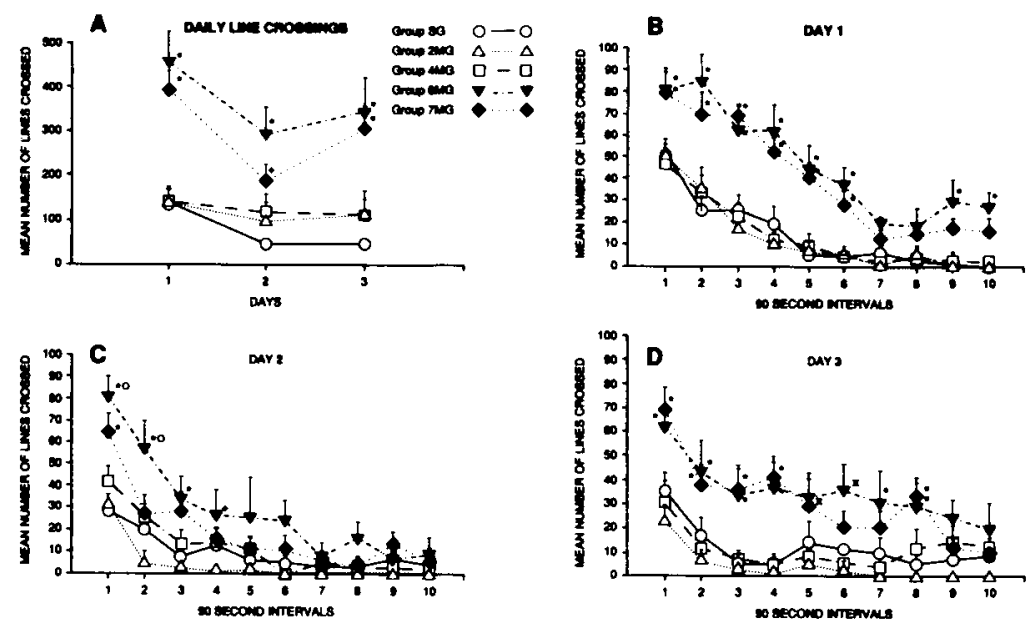

Figure 5. (A) Mean number of lines crosed each day by the rats in the groups included in Experiment 2. * = differs from Groups 2MG, 4MG, and SG $(p<.05)$; $+=$ differs from Group SG only $(p<.05)$. (B,C,D) The overall increased activity levels observed in the rats in Groups 6MG and 7MG were primarily attributable to enhanced number of line crossings during the first half of each test session. Statistically significant differences occurred less frequently after the nith s0-sec interval. * = differs from Groups 2MG, 4MG, and SG $(p<.05)$; $0=$ difiers from Group 7MG $(p<.05) ;+=$ differs from Group 2MG $(p<.05) ; x=$ differs from Groups 2MG and 4MG $(p<.05)$. 
(Group 4MG). Groups 6MG and 7MG never differed from each other.

An analysis of daily open-field activity in 90 -sec time intervals indicated a significant overall effect of treatment for each of the 3 days $[F(4,45)=12.15,5.84$, and 7.06 , $p<.01]$. The effect of time period within each of the three sessions was also significant for each day $[F(9,405)=74.02,39.04,16.31, p<.01]$. As can be seen in Figure 5, each group decreased activity during the course of each daily testing session. However, the decreases were not parallel among the groups for the first 2 days and significant interaction effects were found $[F(36,405)=2.32,2.09, p<.01]$. The interaction was not significant for the third day. The results of multiple comparison tests are indicated in Figures 5B, 5C, and 5D. In sum, these tests indicated that the two lower dosages of TMT had virtually no effect on activity, whereas the two higher dosages increased activity, especially during the first half of each daily session.

Significant group $[F(4,45)=9.08, p<.01]$ and days $[F(2,90)=12.87, p<.01]$ effects were found when the number of rearings was analyzed. Multiple comparison tests indicated that the two higher doses of TMT significantly increased the number of rearings. However, the number of rearings decreased from Day 1 to Day 2 in all groups and the interaction term was not significant.

Effects of TMT on grooming in the open field. The consequences of TMT administration on grooming differed from those produced by hippocampal lesions. A significant group effect was found when the data for time to exhibit the first grooming component following placement into the open field were analyzed $[F(4,45)=2.96$, $p<.05]$. The days effect was not statistically significant, but the interaction effect was $[F(8,90)=4.39, p<.01]$. The pattern of significance revealed by post hoc multiple comparison tests did not present an obvious dose-response-time relationship (see Figure 6A). The rats that were given $2 \mathrm{mg} / \mathrm{kg}$ of TMT (Group 2MG) took significantly longer to begin grooming than those in any other group on the second and third days of testing. The only other significant difference among groups was between Groups 6MG and SG on Day 3. The significant interaction effect is attributable to the relative lack of change exhibited by Group SG over the 3 days, compared with the large increase in latency shown by Group $2 \mathrm{MG}$ and the decreases in latency shown by Groups $4 \mathrm{MG}$ and $6 \mathrm{MG}$ over the 3 days.

A significant group effect was also found when the time sperit grooming was analyzed $[F(4,45)=2.76, p<.05]$. The days and interaction terms were not significant. As was the case for the latency to groom, the multiple comparison tests did not indicate any clear dose-response relationship (Figure 6B). Group 4MG was the only group to differ significantly from any other group; it differed from Groups 2MG and 7MG on Days 1 and 2, and from Group 6MG on Day 1.

Group 4MG continued to differ from the groups that were given both lower and higher doses of TMT when the total number of grooming episodes emitted in the open field was analyzed (Figure 6C). A significant overall groups effect emerged $[F(4,45)=3.21, p<.05]$. Multiple comparison tests indicated that Group $4 \mathrm{MG}$ had more grooming bouts (both complete and fragmented) in the open field than Group 2MG on Days 2 and 3, more than Group 6MG on all 3 days, and more than Group 7MG on Days 1 and 3. Additionally, Group 6MG had fewer grooming bouts than Groups SG and 2MG on Day 1.

No significant effects were found for any of the other measures of grooming taken in the open field.

Effects of TMT on grooming after being sprayed. No significant effects were found when the data for latency to begin grooming after the final spray were analyzed. Significant group $[F(4,45)=2.67, p<.05]$, days $[F(2,90)=8.5, p<.01]$, and interaction $[F(8,90)=$ $2.96, p<.01]$ terms all emerged when the ANOVA was applied to the time spent grooming after being sprayed. Multiple comparison tests indicated that Group 4MG differed from all the groups (Figure 6D) on Day 3 and from Group 7MG on Day 1. Group 7MG also differed from Group 2MG on Day 1.

Statistically significant group $[F(4,45)=4.59, p<.01]$ and days $[F(2,90)=4.65, p<.01]$ terms were found for the number of grooming episodes emitted after spraying. The number of episodes decreased across days (Figure $6 \mathrm{E}$ ) and the group $\times$ days interaction was not significant. Multiple comparison tests indicated that Group 4MG had more grooming episodes than Group SG on the third day, whereas Group 6MG had fewer grooming episodes than Group 4MG on Days 1 and 3. Group 6MG also had fewer grooming episodes than Group SG and fewer than Group 2MG on Days 1 and 2. Group 7MG was lower than these same two groups on the first day and than Group 4MG on Days 1 and 3.

Differences also emerged in number of complete grooming chains emitted after spraying. Both the group $[F(4,45)=3.23, p<.05]$ and days $[F(2,90)=4.88$, $p<.01]$ effects were significant. The groups $\times$ days interaction did not achieve a putative level of statistical significance, although not all groups decreased the number of complete grooming chains across the 3 days (Figure 6F). Multiple comparisons indicated that Group 6MG showed fewer complete grooming chains than Group 2MG on Days 1 and 2, and fewer than Group 4MG on Days 1 and 3. Group 7MG had fewer complete chains than Groups 2MG and 4MG on Day 3.

No statistically significant effects were found when the data from number of fragmented grooming chains or number of components per grooming episode were analyzed.

Histology. An inspection of the slides from all of the rats given TMT indicated that the most noticeable effects of the toxin were on the CA1 and CA3 fields of the septal (dorsal) part of the hippocampus. As can be seen in Figure 7, the two higher doses of the toxin destroyed virtually all of subfield $\mathrm{CA} 3 \mathrm{c}$ and thinned $\mathrm{CA} 1$. The effects of the 4-mg/kg dose of TMT were less apparent. However, pyknotic neurons could be observed within Am- 

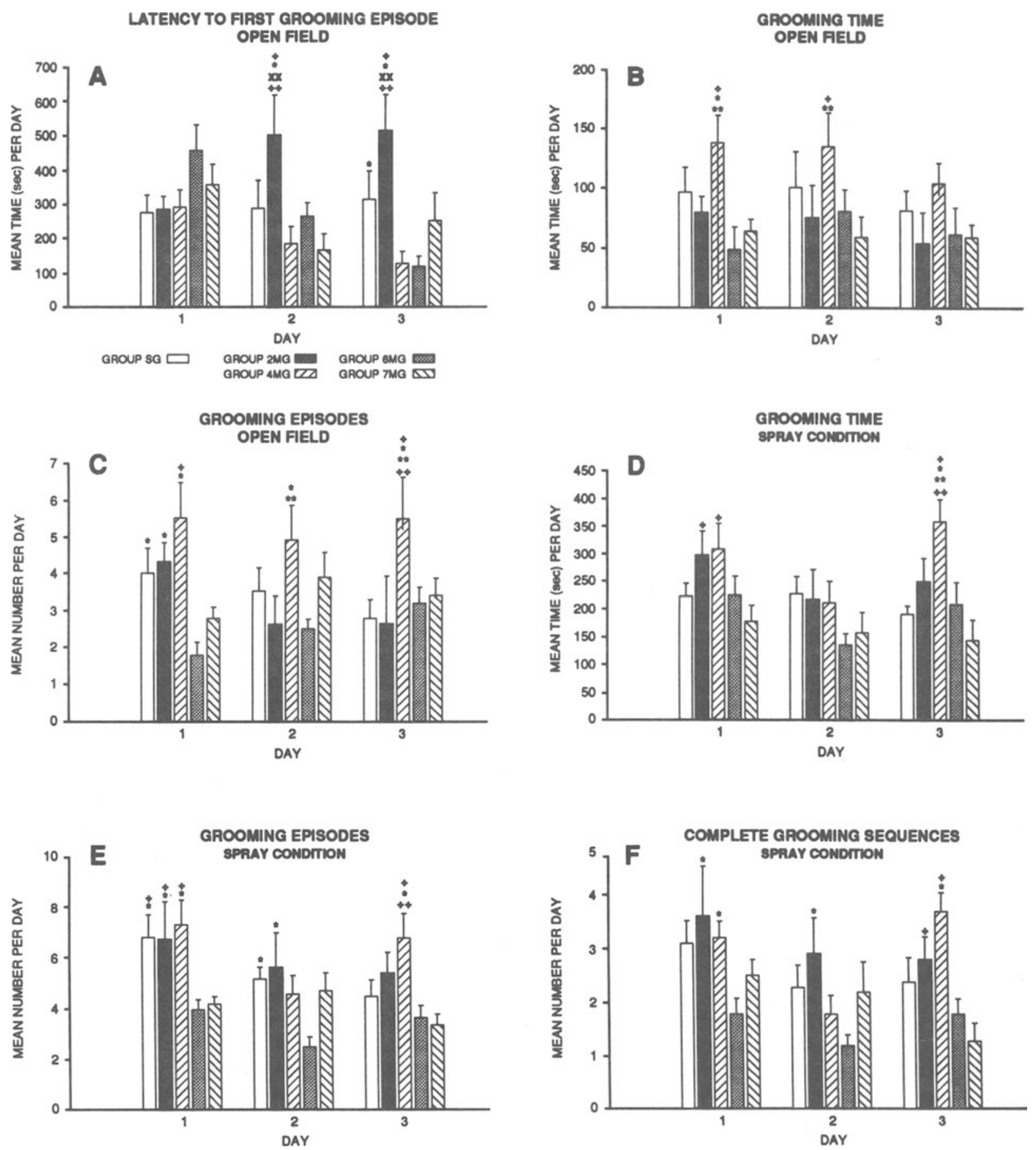

Figure 6. The pattern of TMT effects on the various parameters of grooming did not exhibit a dose-response relationship. A dose of $2 \mathrm{mg} / \mathrm{kg}$ of the toxin significantly increased latency to groom in the open field, compared with all other groups (A), but $4 \mathrm{mg} / \mathrm{kg}$ produced the only significant effect on mean grooming time (B) and number of grooming episodes emitted in the open field (C). After being sprayed with the sucrose solution, Group 4MG also demonstrated more grooming time during the final observation period (D). However, the lower doses of TMT did not change the total number of grooming episodes (E), or the number of complete grooming sequences (F), as compared with the control group (Group SG), but the higher doses tended to decrease these two measures. * = differs from Group $6 \mathrm{MG}$ $(p<.05) ;+=$ differs from Group 7MG $(p<.05) ; * *=$ differs from Group $2 M G(p<.05) ;++=$ differs from Group SG $(p<$ $.05) ; x \times=$ differs from Group 4MG $(p<.05)$. 

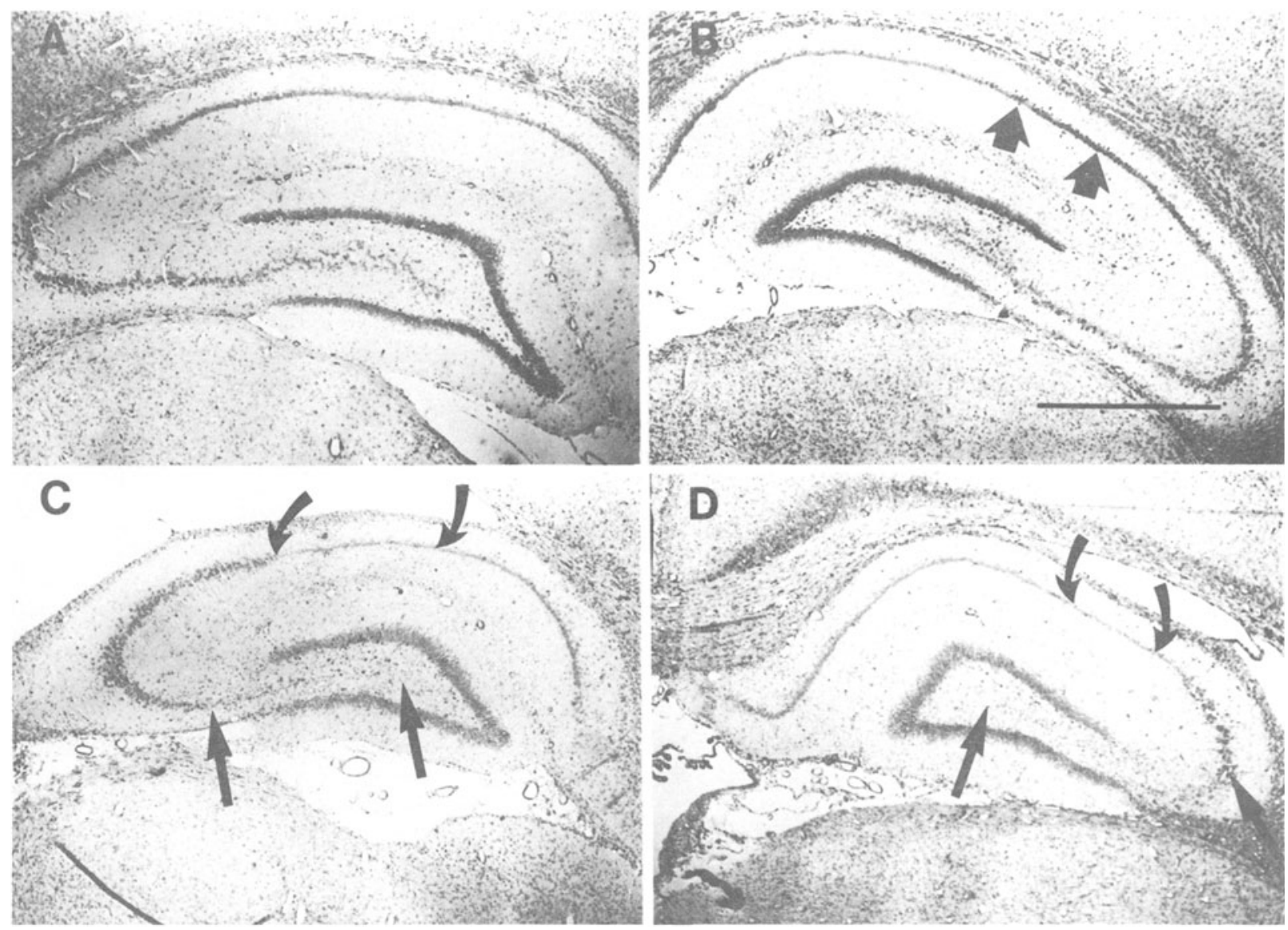

Figure 7. Photomicrographs of representative coronal sections through the brains of rats given $2 \mathrm{mg} / \mathrm{kg}$ (A), $4 \mathrm{mg} / \mathrm{kg}$ (B), $6 \mathrm{mg} / \mathrm{kg}$ (C), or $7 \mathrm{mg} / \mathrm{kg}$ (D) of TMT. Neuron loss is evident from the CA3c area of Ammon's horn in the rats from Groups 6MG and $7 \mathrm{MG}$ (between straight arrows, panels $\mathrm{C}$ and $\mathrm{D}$ ) and the thickness of $\mathrm{CA1}$ is reduced when this sector (between curved arrows in panels $\mathrm{C}$ and D) is compared with the same region in a section taken through the dorsal hippocampus of a rat in Group 2MG (A). Although cell loss is not evident upon inspection of the histology from the rats in Group $4 \mathrm{MG}$, many neurons within areas of the hippocampus showing cell loss after higher doses of TMT are pyknotic after $4 \mathrm{mg} / \mathrm{kg}$ of TMT (examples between arrowheads in panel B). Magnification bar $=$ $1.1 \mathrm{~mm}$ and represents all micrographs.

mon's horn and the dentate gyrus and analyses of the quantitative data indicated that the $4-\mathrm{mg} / \mathrm{kg}$ dose significantly reduced the number of neurons in medial frontal and pyriform cortical samples, as well as fields CA1 and CA3 of Ammon's horn (see Figure 8). The two higher doses of TMT reduced cell numbers in all areas from which measurements were taken, but the $2-\mathrm{mg} / \mathrm{kg}$ dose only produced a statistically significant effect in field CA1 of Ammon's horn (Figure 8).

To determine whether the number of neurons counted in each area in the rats from the TMT-treated groups might predict behavioral effects, simple regressions were conducted on the various behavioral variables on the cell counts that had indicated significant differences from normal. The only statistically significant effects were the activity of the rats in Group 6MG regressed upon the cell count for CA3 as a predictor $\left[r^{2}=.40, F(1,9)=5.33\right.$, $p<.05]$ and on the cell count in the pyriform cortex as a predictor $\left[r^{2}=.47, F(1,9)=7.01, p<.05\right]$.
Multiple regressions were also conducted, using the sum of all lines crossed over all 3 days for each rat and regressed for each treatment group on cell counts from all six anatomical areas as predictors, but no significant effects were found. Furthermore, no significant regression values were found for rearing scores or for any of the grooming measures.

\section{DISCUSSION}

The main purpose of the present study was to determine the effects of hippocampal lesions on grooming in the rat and to compare these effects with those produced by exposure to trimethyltin. However, before discussing the effects of the treatments on grooming, some consideration will be given to the consequences of the lesions or the toxin on open-field activity.

Perhaps the most comprehensive explanation for the increased open-field activity exhibited by rats with hippo- 

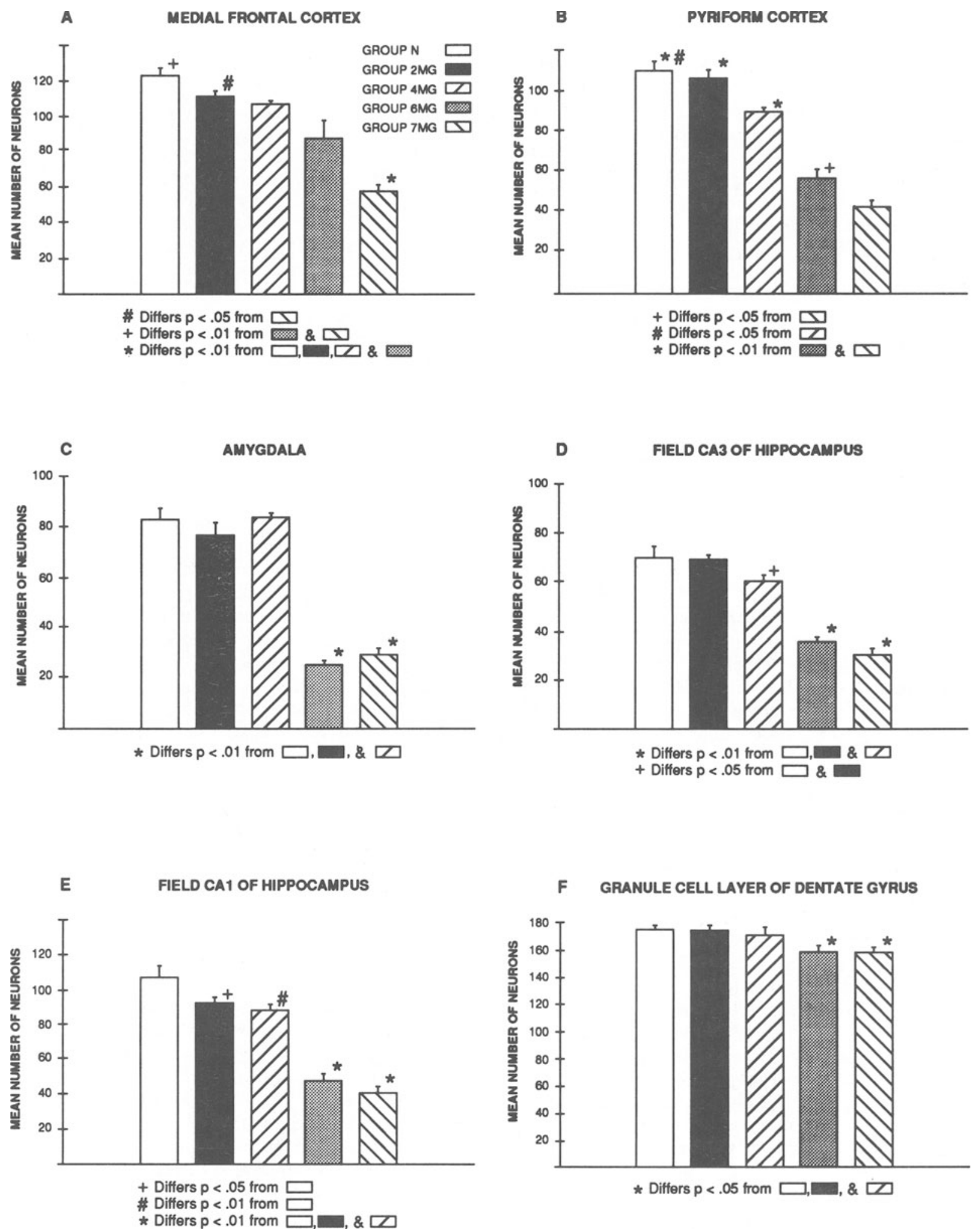

Figure 8. The $6-$ and 7-mg/kg doses of TMT significantly decreased the number of neurons in each of the areas examined. However, relative to normal controls, the $4-\mathrm{mg} / \mathrm{kg}$ dose significantly decreased neuron numbers only in pyriform cortex (B) and in the CA1 and CA3 subfields of the hippocampus (D and E). The $2-\mathrm{mg} / \mathrm{kg}$ dose of TMT only produced a significant effect in hippocampal subfield CA1 (E). 
campal lesions has been offered by O'Keefe and Nadel (1978, pp. 253-260), who argued for two primary causes of hippocampal lesion-induced hyperactivity-decreased fear of novelty and increased reactivity (presumably without emotional valence) to stimuli. Although the present experiments were not designed to test these two hypotheses, some of the data may be relevant to their evaluation. For example, it does not appear that the pattern of activity emitted by the rats in Group HL is particularly compatible with the hypothesis that these rats are inevitably less fearful in a novel environment. Group HL was significantly less active than either cortically lesioned or normal control rats during the first $1.5 \mathrm{~min}$ in the open field on the first day of testing. Furthermore, although the total number of lines crossed by the rats in Group HL decreased significantly across the 3 days of testing, the mean number crossed by this group during the first $90 \mathrm{sec}$ was doubled on Days 2 and 3, compared with Day 1. Assuming, as O'Keefe and Nadel do, that initial immobility in a novel environment is an indicator of fear, these observations may be interpreted to mean that the rats in Group HL were actually more fearful during their initial exposure to the open field and that enhanced activity emerged only when the initial reaction subsided.

The hyperactivity exhibited by Groups 6MG and 7MG is consistent with previous reports (Ruppert, Walsh, Reiter, \& Dyer, 1982; Swartzwelder, Dyer, Holahan, \& Myers, 1981; Woodruff et al., 1988). The activity pattern shown by these two groups of TMT-treated rats differed slightly from that of Group HL in that the initial, short-lived activity reduction was absent. The cause of this difference can only be suggested, but a series of studies by Jarrard and his colleagues (reviewed by Jarrard, 1986) have indicated that aspiration lesions of the hippocampus produce a different pattern of behavioral effects than that produced by either mechanical or neurotoxic (e.g., intrahippocampal injection of ibotenic acid or colchicine) damage, which is restricted to various components of the hippocampal formation. Thus, it is possible that TMT is more selective in its destruction of the hippocampus than an aspiration lesion is, and this may account for the difference in activity pattern early in training. This proposal could be tested as a hypothesis using intrahippocampal microinjections of ibotenic acid or colchicine, particularly into CA3, because destruction of this region of Ammon's horn was found to predict a significant part of the activity change in the 6MG TMT group. However, the regression analysis indicated that lower cell numbers in the pyriform cortex also predicted increased activity. Therefore, it may be that a combination of hippocampal and pyriform destruction induced by TMT prevented the significant initial short-term activity reduction observed in the rats with hippocampal lesions.

Despite the differences in activity patterns during the first minutes of testing, the effects of TMT on open-field activity are like those produced by hippocampal lesions. Furthermore, although the dose-response curve for TMTinduced changes in activity in the present paradigm is steep and is essentially asymptotic, the dose-response relationship is as might be expected, that is, larger doses produce a larger effect. Finally, even with the relatively limited stereological data collected in the present study, loss of neurons from the hippocampus and pyriform cortex appear to be related to increased open-field activity. However, when grooming is evaluated, the dose-response relationship does not even suggest a linear function and cell loss patterns do not hint that a loss of neurons in the hippocampus is responsible for TMT-induced changes in this behavioral measure. For these reasons, the effects of hippocampal lesions on grooming and the effects of TMT on grooming will be discussed separately.

Previous reports indicate that hippocampal lesions do not alter grooming of animals in their home cages (Glickman, Higgins, \& Isaacson, 1970; Kim et al., 1970). However, hippocampal lesions do decrease grooming in rats that are placed in a novel environment, such as a glass observation tank or an open-field arena (Bär, Gispen, \& Isaacson, 1981; Oades \& Isaacson, 1977; Reinstein, Hannigan, \& Isaacson, 1982). The results of the present study also show a decrease in grooming by rats with hippocampal lesions in a novel environment and add to the previous observations by indicating that the reduction is specifically attributable to a reduction in the number of complete grooming chains during the first 2 days of testing, as compared with the number of complete chains produced by normal rats or rats with neocortical lesions. Because hippocampal lesions do not affect grooming in familiar environments, the explanation for the hippocampal lesion-induced reduction in number of complete grooming chains would appear to be related to the responses of Group HL to the novel environment.

Grooming may subserve several functions in addition to the obvious role in cleaning the body surface; it may aid in thermoregulation, or be used in social signaling. Grooming may also be used for self-stimulation by pregnant females to promote milk production (Sachs, 1988). None of these functions, however, seem to explain the enhancement of grooming in novel environments, and grooming in such a context is typically regarded as an example of displacement behavior (e.g., Fentress, 1988). Displacement behaviors are allochotonous, that is, unrelated to the drives that normally evoke them, and may be expressed when competing behavioral tendencies are strongly activated and in conflict. A third behavioral pattern is thereby disinhibited (Fentress, 1986; Reynierse, Scavio, \& Ulness, 1970). The competing tendencies, when the rat is placed into the open field by the experimenter, may be reactions to a situation having predatory overtones (Suarez \& Gallup, 1981). These reactions would include behaviors such as immobility and would conflict with a tendency to explore a new environment ( $O$ 'Keefe \& Nadel, 1978, pp. 234-255). The consequence of the conflict is enhanced neural activation and the emergence of grooming. In most situations, hippocampal damage appears to lessen exploration and may decrease fear (O'Keefe \& Nadel, 1978), thus reducing the substrates 
that produce the displacement behavior of grooming. The neurobiological basis of this effect of hippocampal lesions may be the loss of hippocampal input to the nucleus accumbens, resulting in changes in dopaminergic activity within this structure (Reinstein et al., 1982).

There was a tendency for the rats in Group HL to be less likely than either of the control groups to groom after spray, as was evidenced by the significantly longer latencies to groom on Days 2 and 3 and also in the reduction in number of grooming components per episode compared with Group $\mathbf{N}$ during Days 1, 2, and 3 and Group CL on Day 3. Additionally, Group HL groomed for a shorter period of time than Group CL did on each day of testing and for a shorter period than Group $\mathrm{N}$ on Day 3. These observations are compatible with the previous results discussed above and may be attributable to the loss of hippocampal input to the nucleus accumbens (Reinstein et al., 1982).

Although cortical lesions appeared to have little effect on grooming in the open field, the rats in Group CL differed significantly from those in Groups $\mathrm{N}$ and $\mathrm{HL}$ in several measures of grooming after spray; this confounds the interpretation of the effects of hippocampal lesions. The cortical lesions appear to increase grooming, at least using some measures of the response. Group $\mathrm{CL}$ groomed longer than Group N on Day 1 and than Group HL on all 3 days. Group CL also emitted more grooming episodes than Group $\mathbf{N}$ on all 3 days. This is probably due to the significant increase in number of fragmented grooming sequences. Similar effects were also found for Group HL, but these could be attributed to the cortical damage done in the course of removing the hippocampus.

Grooming after being sprayed with sugar water is most likely a body surface maintenance response. Lesions restricted to lateral sensorimotor cortex have not been reported to produce substantial effects on grooming, even when rats are observed after being immersed in a tank of water (e.g., Vanderwolf et al., 1978). However, the presence of the sucrose in the spray presumably provides a stronger and more enduring stimulus for elicitation of grooming than water alone (Moore et al., 1987). The present data suggest that in the presence of such an irritant, the intact dorsolateral sensorimotor cortex participates in the modulation of the brainstem mechanisms that are responsible for organizing the chain of behavioral components that comprise grooming, such that the total sequences are performed.

It is noteworthy that the effects of TMT on grooming did not mimic those produced by the surgical lesions. This observation is important because it indicates that administration of TMT does not always produce the same behavioral effects that hippocampal lesions do. An analysis of the effects of TMT on grooming indicated that the 2and $4-\mathrm{mg} / \mathrm{kg}$ doses produced significant changes compared with controls in several measures of this complex behavior, whereas the rats that were given the higher doses of the toxicant failed to differ from the controls on these same measures. These results appear counter- intuitive and also fail to correlate with the measures of neuropathology made in the present study. Furthermore, most reports of TMT effects on behavior indicate the expected dose-response relationship to be an increasing behavioral deficit with increasing doses of TMT (e.g., Bushnell, 1990; McMillan \& Wenger, 1985). However, an exception to this general trend is provided by a series of experiments by Messing, Sparber, and their co-workers (Cohen, Messing, \& Sparber, 1987; Gerbec, Messing, \& Sparber, 1988; Messing \& Sparber, 1986). The doses used by this group of investigators were 3,6 , and $7.5 \mathrm{mg}$ of TMT $/ \mathrm{kg}$ rat bodyweight. Cohen et al. (1987) used a paradigm that autoshaped rats to touch a lever, which extended from the wall of an operant chamber, to study the effects of delay of reinforcement on learning. Two delays, 4 and $6 \mathrm{sec}$, were used. TMT-induced behavioral effects were found only at $6 \mathrm{sec}$, and the rats that were given 3 or $6 \mathrm{mg} / \mathrm{kg}$ of TMT differed from the controls, but the rats that were given $7.5 \mathrm{mg} / \mathrm{kg}$ did not. Additional studies found changes in two brain systems that appear to parallel the effects of TMT on the autoshaped leverpressing. Messing and Sparber (1986) reported increased binding of the $\beta$-adrenergic ligand dihydroalprenol in frontal cortex and a combination of amygdala and pyriform cortex in rats that were given $6 \mathrm{mg} / \mathrm{kg}$ of TMT, but not in rats that were given $7.5 \mathrm{mg} / \mathrm{kg}$. This suggests that median doses of TMT might alter noradrenergic transmission in these two areas and it may be that this change in these two brain regions is related to the behavioral effects observed by Cohen et al. and in the present experiment. A study by Gerbec et al. (1988) also implicates TMTinduced changes in corticosterone binding in rat hippocampus in nonlinear dose-behavior response effects of TMT. Gerbec et al. used a fixed-ratio (FR) operant schedule and doubled the response requirement daily until an FR 128 was reached. The control rats demonstrated maximum response rates when FR16 and FR32 schedules were in effect. The rats that were given $6 \mathrm{mg} / \mathrm{kg}$ of TMT responded less than controls when the FR15 and FR32 schedules were in effect, but did not differ when other ratio requirements obtained. The rats that were given $7.5 \mathrm{mg} / \mathrm{kg}$ of TMT responded more than the controls when lesser ratio requirements produced lower control response rates, but were similar to the controls when FR16 and FR32 requirements were in place. Corticosterone binding to hippocampal cytostolic protein was significantly reduced in the rats that were given $6 \mathrm{mg} / \mathrm{kg}$ of TMT, compared with the rats that were given $7.5 \mathrm{mg} / \mathrm{kg}$ of TMT, although the higher dose destroyed more of the hippocampus.

Therefore, both the present study and those from other laboratories indicate that the behavioral effects produced by TMT may be more complex than those that might be predicted from a simple evaluation of frank neuropathology. Although many behavioral effects may be primarily and parametrically related to TMT-induced destruction of hippocampal neurons (e.g., Bushnell, 1990), other behavioral effects of TMT will need to be explained by fur- 
ther studies that attempt to relate changes in intact neuronal systems to alterations in specific behavioral paradigms.

\section{REFERENCES}

Balaban, C. D., O'Callaghan, J. P., \& Billingsley, M. L. (1988). Trimethyltin-induced neuronal damage in the rat brain: Comparative studies using silver degeneration stains, immunocytochemistry and immunoassay for neuronotypic and gliotypic proteins. Neuroscience, 26, 337-361.

BÄR, P. R., GISPEN, W. H., \& ISAACSON, R. L. (1981). Behavioral and regional neurochemical sequelae of hippocampal destruction in the rat. Pharmacology, Biochemistry \& Behavior, 14, 305-312.

Berridge, K. C. (1989). Progressive degradation of serial grooming chains by descending decerebration. Behavioural Brain Research, 33, 241-253.

Brown, A. W., Aldridge, W. N., Street, W. B., \& Verschoyle, R. D. (1979). The behavioral and neuropathological sequelae of intoxication by trimethyltin compounds in the rat. American Journal of Pathology, 97, 59-76.

BUSHNELL, P. J. (1990). Delay-dependent impairment of reversal learning in rats treated with trimethyltin. Behavioral \& Neural Biology, 54, 75-89.

Chang, L. W. (1986). Neuropathology of trimethyltin: A proposed pathogenetic mechanism. Fundamental \& Applied Toxicology, 6, 217-232.

Cohen, C. A., Messing, R. B., \& Sparber, S. B. (1987). Selective learning impairment of delayed reinforcement autoshaped behavior caused by low doses of trimethyltin. Psychopharmacology, 93, 301-307.

Colburn, D., IsaAcson, R. L., Bohus, B., \& Gispen, W. H. (1977). Limbic-midbrain and ACTH-induced excessive grooming. Life Sciences, 21, 393-402.

Coluins, V. J. (1976). Principles of anesthesiology (2nd ed.). Philadelphia: Lea \& Febiger.

Dyer, R. S., Deshields, T. L., \& Wonderlin, W. F. (1982). Trimethyltin-induced changes in gross morphology of the hippocampus. Neurobehavioral Toxicology \& Teratology, 4, 141-147.

Dyer, R. S., Walsh, T. J., WonderLin, W. F., B Bercegeay, M. (1982). The trimethyltin syndrome in rats. Neurobehavioral Toxicology \& Teratology, 4, 127-133.

Fentress, J. C. (1986). Ethology and the neural sciences. In R. Campan \& R. Zayan (Eds.), Relevance of models and theories in ethology (pp. 77-107). Toulouse: Privat.

FENTRESs, J. C. (1988). Expressive contexts, fine structure, and central mediation of rodent grooming. Annals of the New York Academy of Sciences, 525, 18-26.

Gallup, G. C., JR. (1974). Animal hypnosis: Factual status of a fictional concept. Psychological Bulletin, 81, 836-853.

Gerbec, E. N., Messing, R. B., \& Sparber, S. B. (1988). Parallel changes in operant behavioral adaptation and hippocampal corticosterone binding in rats treated with trimethyltin. Brain Research, $\mathbf{4 6 0}$, 346-351.

Guckman. S. E., Higgins, T. J., IsaAcson, R. L. (1970). Some effects of hippocampal lesions on behavior of mongolian gerbils. Physiology \& Behavior, 5, 931-938.

Hannigan, J. H., JR., Springer, J. E., \& Isacson, R. L. (1984). Differentiation of basal ganglia dopaminergic involvement in behavior after hippocampectomy. Brain Research, 291, 83-91.

IsAacson, R. L. (1982). The limbic system (2nd ed.). New York: Plenum.

IsAACSON, R. L., WoodrufF, M. L. (1975). Spontaneous alternation and passive avoidance behavior in rats after hippocampal lesions. In B. L. Hart (Ed.), Experimental Psychobiology (pp. 102-109). San Francisco: Freeman.

JARRARD, L. E. (1986). Selective hippocampal lesions and behavior: Implications for current research and theorizing. In R. L. Isaacson \& K. H. Pribram (Eds.), The hippocampus (Vol. 3, pp. 93-127). New York: Plenum.
KIm, C., Choi, H., Kim, J. K., Chang, H. K., PARK, R. S., \& KANG, I. Y. (1970). General behavioural activity and its component patterns in hippocampectomized rats. Brain Research, 19, 379-394.

KolB, B., \&onneman, A. J. (1974). Frontolimbic lesions and social behavior in the rat. Physiology \& Behavior, 13, 637-643.

MCMillan, D. E., \& Wenger, G. R. (1985). Neurobehavioral toxicology of trialkyltins. Pharmacological Reviews, 37, 365-379.

Messing, R. B., \& Sparber, S. B. (1986). Increased forebrain $\beta$ adrenergic ligand binding induced by trimethyltin. Toxicology Letters, 32, 107-112.

Moore, H. D., Low, C. A., Kulkosky, P. J. (1987). Grooming: The effects of sprayed sapid irritants. Proceedings of the NIH Centennial MBRS-MARC Symposium, 15, 99.

Nonneman, A. J., KolB, B. B. (1974). Lesions of hippocampus or prefrontal cortex alter species-typical behaviors in the cat. Behavioral Biology, 12, 41-54.

OADES, R., \& ISAACSON, R. L. (1977). p-Chlorophenylalanine-produced effects on behavior in intact and brain-damaged rats. Behavioral Biology, 20, 500-506.

OKEEFE, J., \& NADEL, L. (1978). The hippocampus as a cognitive map. Oxford: Oxford University Press.

Pellegrino, L. J., Pellegrino, A. S., Cushman, A. J. (1979). A stereotaxic atlas of the rat brain (2nd ed.). New York: Plenum.

Reinstein, D. K., Hannigan, J. H., JR., IsaAcson, R. L. (1982). Time course of certain behavioral changes after hippocampal damage and their alteration by dopaminergic intervention into nucleus accumbens. Pharmacology, Biochemistry \& Behavior, 17, 193-202.

Reynierse, J. H., Scavio, M. J., JR., Ulness, J. D. (1970). An ethiological analysis of classically conditioned fear. In J. H. Reynierse (Ed.), Current issues in animal learming: A colloquium (pp. 33-54). Lincoln: University of Nebraska Press.

Richmond, G., SACHs, B. D. (1980). Grooming in Norway rats: The development and adult expression of a complex motor pattern. Behaviour, 75, 82-96.

RuPPERT, P. H., WAlsh, T. J., Reiter, L. W., \& Dyer, R. S. (1982). Trimethyltin-induced hyperactivity: Time course and pattern. Neurobehavioral Toxicology \& Teratology, 4, 135-139.

SACHS, B. D. (1988). The development of grooming and its expression in adult animals. Annals of the New York Academy of Sciences, 525, $1-17$.

SunRez, S. D., \&allup, G. G., Jr. (1981). Predatory overtones of open-field testing in chickens. Animal Learning \& Behavior, 9 , 153-163.

Swartzwelder, H. S., Dyer, R. S., Holahan, W., \& Myers, R. D. (1981). Activity changes in rats following acute trimethyltin exposure. Neurotoxicology, 2, 589-593.

VANDerwolf, C. H., Kolb, B., \& CoOley, R. K. (1978). Behavior of the rat after removal of the neocortex and hippocampal formation. Journal of Comparative \& Physiological Psychology, 92, 156-175.

WoOdRuFF, M. L., BAILEY, S. D. (1979). Hippocampal lesions and immobility responses in the rat. Physiological Psychology, 7, 254-258.

Woodruff, M. L., Baisden, R. H., Whittington, D. L., \& BensoN, A. E. (1987). Embryonic hippocampal grafts ameliorate the deficit in DRL acquisition produced by hippocampectomy. Brain $R e-$ search, 408, 97-117.

Woodruff, M. L., Baisden, R. H., Whittington, D. L., Shelton, N. L., \& WRAY, S. (1988). Grafts containing fetal hippocampal tissue reduce activity and improve passive avoidance in hippocampectomized or trimethyltin-exposed rats. Experimental Neurology, 102, 130-143.

Woodruff, M. L., Hatton, D. C., \& MeYER, M. E. (1975). Hippocampal ablation prolongs immobility response in rabbits (Oryctolagus cuniculus). Journal of Comparative \& Physiological Psychology, 88, 329-334.

(Manuscript received May 31, 1991; revision accepted for publication June 17,1992 .) 